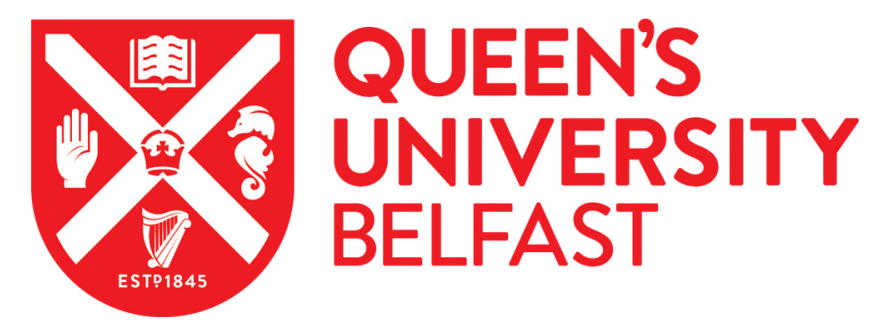

\title{
Optimising the visibility of graphene and graphene oxide on gold with multilayer heterostructures
}

Velický, M., Hendren, W. R., Donnelly, G. E., Katzen, J. M., Bowman, R. M., \& Huang, F. (2018). Optimising the visibility of graphene and graphene oxide on gold with multilayer heterostructures. Nanotechnology, 29(27), 275205. https://doi.org/10.1088/1361-6528/aabec1

Published in:

Nanotechnology

Document Version:

Peer reviewed version

Queen's University Belfast - Research Portal:

Link to publication record in Queen's University Belfast Research Portal

Publisher rights

(C) 2018 IOP Publishing Ltd.

This work is made available online in accordance with the publisher's policies. Please refer to any applicable terms of use of the publisher.

\section{General rights}

Copyright for the publications made accessible via the Queen's University Belfast Research Portal is retained by the author(s) and / or other copyright owners and it is a condition of accessing these publications that users recognise and abide by the legal requirements associated with these rights.

Take down policy

The Research Portal is Queen's institutional repository that provides access to Queen's research output. Every effort has been made to ensure that content in the Research Portal does not infringe any person's rights, or applicable UK laws. If you discover content in the Research Portal that you believe breaches copyright or violates any law, please contact openaccess@qub.ac.uk. 


\title{
Enhanced visibility of graphene and graphene oxide
}

\section{mono/multi-layers on thin gold}

\author{
Matěj Velický,* William R. Hendren, Gavin E. Donelly, Joel M. Katzen, Robert M. \\ Bowman, and Fumin Huang*
}

Centre for Nanostructured Media, School of Mathematics and Physics, Queen's University Belfast, University Road, Belfast, BT7 1NN, UK

* To whom correspondence should be addressed. Tel: +44 (0)28 9097 2503; e-mail:

f.huang@qub.ac.uk or matej.velicky@gmail.com.

Keywords: visibility, optical contrast, graphene, graphene oxide, Au substrate.

\begin{abstract}
Metals have been increasingly used as substrates in devices based on two-dimensional (2D) materials. However, the high reflectivity of bulk metals results in low optical contrast $(<3 \%)$ and therefore poor visibility of mono- and few-layer transparent 2D materials on these surfaces. Here we demonstrate that by engineering the complex reflectivity of a purposedesigned multilayer heterostructure composed of thin $\mathrm{Au}$ films $(2-8 \mathrm{~nm})$ on $\mathrm{SiO}_{2} / \mathrm{Si}$ substrate, the optical contrast graphene and graphene oxide (GO) can be significantly enhanced in comparison to bulk Au, up to about 3 and 5 times, respectively. In particular, we achieved $\sim 17 \%$ contrast for monolayer GO, the highest to date, and even 2 times higher than that on the bare $\mathrm{SiO}_{2} / \mathrm{Si}$ substrate. The experimental results are in good agreement with theoretical simulations. Although this concept is demonstrated for $\mathrm{Au}$, the methodology is applicable to other metals and can be adopted to design a variety of high-contrast metallic substrates. This will facilitate research and applications of $2 \mathrm{D}$ materials in a broad range of areas, such as plasmonics, photonics, catalysis and sensors.
\end{abstract}




\section{Introduction}

Underlying substrates are critical to the functionality of two-dimensional (2D) materials and have been shown to affect their optical, electrical and chemical properties, as manifested in spectroscopy, electronic transport, and electrochemistry [1-4]. Metals are popular substrates in 2D material-based devices, which are central to a wide range of applications, including photonics, plasmonics, catalysis, and sensors [5-9]. A variety of 2D materials have been grown on various metallic substrates $(\mathrm{Ni}, \mathrm{Cu}, \mathrm{Au})$ by chemical vapour deposition [10-12]. Metals also have been effectively used in doping of $2 \mathrm{D}$ materials to achieve tuneable electronic and optical properties by shifting the Fermi level, which greatly extends their applicability [13, 14]. However, many 2D materials, in particular the highly transparent ones, such as graphene and graphene oxide (GO), are hardly visible on bulk metallic surfaces. Monolayers of these 2D materials have only a modest optical contrast, generally less than $3 \%$, as a consequence of the low light absorption of the transparent 2D material and high reflectivity of the metal surface underneath [15]. This poses a significant practical challenge in device preparation, fundamental research, and technology development. It is therefore essential to develop innovative strategies to improve the optical visibility of 2D materials on metallic surfaces, however, little research effort has been spent in that regard. The majority of past investigations have focused on dielectric substrates such as silicon oxide/nitride or metal oxides [16-19], and only a few studies have considered 2D materials on metal surfaces [15, 20-22].

Here we show that the optical contrast of graphene and GO on metals can be significantly enhanced by engineering the complex reflectivity of the underlying substrate. We demonstrate this on one of the most popular metal: gold. Au is widely used in many optoelectronic devices due to its excellent chemical stability and superior plasmonic, catalytic, and doping properties $[5,6,23]$. Instead of a bulk Au surface, we designed and fabricated heterostructures composed of thin Au films of various thickness $(2.1,4.6,7.5,10.1$, and 13.0 
$\mathrm{nm}$ ) on $93 \mathrm{~nm} \mathrm{SiO} / \mathrm{Si}$ substrates (figure 1), which allows for tuning of the optical properties by varying the thickness of the Au film. Optical contrast of ultrathin $2 \mathrm{D}$ materials is solely determined by the complex reflectivity of the underlying structure [24]. Hence, by purposedesigning the optical properties of the underlying heterostructure, one can achieve extraordinary optical contrast of graphene and $\mathrm{GO}$ on $\mathrm{Au}$. We found that ultrathin $\mathrm{Au}$ films (2 $-8 \mathrm{~nm}$ ) in such stratified heterostructure can remarkably enhance the optical visibility of graphene and GO in comparison to bulk $\mathrm{Au}$ film, by up to 3 and 5 times, respectively. In particular, with $4.6 \mathrm{~nm} \mathrm{Au}$ film, we achieved a maximum positive contrast of $(16.6 \pm 0.4) \%$ and a minimum negative contrast of $-(15.2 \pm 0.5) \%$ for monolayer GO. To our knowledge, this is one of the highest optical contrast achieved to date for GO [25], even 2 times higher than that on the bare $\mathrm{SiO}_{2} / \mathrm{Si}$ substrate. Our experimental results are in excellent agreement with theoretical simulations averaged over the transverse-electric (TE) and transverse-magnetic (TM) polarisations and the range of incident angles, an essential approach when a high numerical aperture (NA) objective is used [15]. The identification of the number of layer of graphene and GO was confirmed by atomic force microscopy (AFM) and Raman spectroscopy.

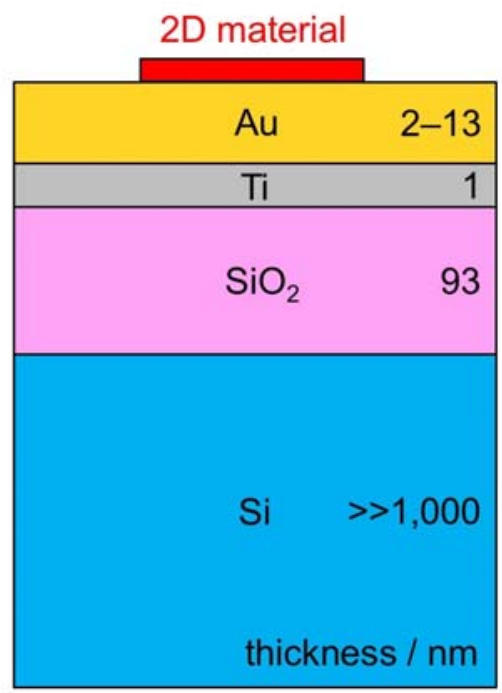

Figure 1 | Schematic of the stratified heterostructures.

\section{Methods}

Fabrication of stratified heterostructures 
All Au films were prepared by physical vapour deposition (Kurt J. Lesker CMS-A DC magnetron sputterer) on $93 \mathrm{~nm} \mathrm{SiO}_{2} / \mathrm{Si}$ diced wafers (or plain $\mathrm{Si}$ for bulk $\mathrm{Au}$ ) (IDB Technologies Ltd). A layer of Ti (1 and $10 \mathrm{~nm}$ for thin and bulk Au, respectively) was deposited first to increase adhesion of $\mathrm{Au}$ to the substrate. Contamination of 2D materials with airborne molecules is an issue affecting many research areas [26-29]. To minimise contamination at the 2D material/Au interface, graphene was mechanically exfoliated using the scotch-tape method [30] from bulk graphite (NGS Naturgraphit $\mathrm{GmbH}$ ) onto the freshly-prepared Au films within 2 min of their exposure to air, producing high-quality, clean, and uniform crystals. GO flakes were drop-cast on the Au films from an aqueous solution ( $>95 \%$ monolayer, Graphenea Inc) diluted to $0.4 \mu \mathrm{g} \mathrm{mL}{ }^{-1}$.

\section{Au film characterisation}

ESM-300 ellipsometer (J.A. Woollam Co, Inc) was used to determine the optical constants $n$ (real part of refractive index) and $\kappa$ (extinction coefficient) of Au and Ti by Complete EASE 5.0 software (J.A. Woollam Co, Inc) as shown in Suppl. figure S1. X-ray reflectometry (XRR), carried out using a Bruker D8 Discover diffractometer, DIFFRAC.Measurement Center, and Leptos 7.8 software, were used to determine the thicknesses of Au and Ti layers (Suppl. figure S2).

\section{Characterisation of 2D materials}

Quality and thickness of 2D materials were assessed by optical microscopy, AFM and Raman spectroscopy. Optical images were taken using a BX51 microscope (Olympus Corp) with an Infinity2-2 CCD camera and Infinity Capture 6.2 software (Lumenera Corp). AFM was performed using Digital Instruments Veeco Dimension 300 in a tapping mode with $30 \mathrm{~nm} / 80$ $\mathrm{kHz} / 4.5 \mathrm{~N} \mathrm{~m}^{-1}$ Si-SPM tips (Nanosensors ${ }^{\mathrm{TM}}$ ). Raman spectra were measured with a $532 \mathrm{~nm}$ excitation laser of $1.0 \mathrm{~mW}$ power, focused through the $100 \times$ objective to $\sim 1 \mu \mathrm{m}^{2}$ spot size and collected through a back-reflection configuration by a Jobin Yvon HR640 Raman spectrometer 
operated by Andor MCD 2.6 software. Two-point probe was used to measure the resistance and estimate resistivity of the $\mathrm{Au}$ films (not taking into account the thickness of the discontinuous Ti sublayer).

\section{Optical contrast measurement}

Two different methods, optical reflectance spectroscopy and optical microscopy with a selection of bandpass filters (10 $\mathrm{nm}$ bandwidth), were used to determine the optical contrast of the $2 \mathrm{D}$ materials. The contrast spectrum, $C(\lambda)$ in percentage $(\%)$, was determined from the reflectance measurements by a QE65 spectrometer and SpectraSuite software (Ocean Optics Inc) using the following formula:

$$
C(\lambda)=\frac{R_{\mathrm{S}}(\lambda)-R_{\mathrm{m}}(\lambda)}{R_{\mathrm{S}}(\lambda)} \cdot 100 \%
$$

where $R_{\mathrm{s}}(\lambda)$ and $R_{\mathrm{m}}(\lambda)$ are the wavelength-dependent reflectance of the substrate and 2D material, respectively (examples of the reflectance spectra are shown in Suppl. figure S3). All reflectance spectra were collected using a 50× objective (Olympus MPLN50xBD, NA = $0.75, \sim 4 \mu \mathrm{m}^{2}$ spot-size), with the exception of figures $3 \mathrm{c}, 5 \mathrm{c}$, and Suppl. figure $\mathrm{S} 3$, where the $100 \times$ objective (Olympus MPLN100xBD, NA $=0.9, \sim 0.8 \mu \mathrm{m}^{2}$ spot-size) was used.

The optical contrast was also determined from optical micrographs $(50 \times$ objective) taken with $10 \mathrm{~nm}$ bandwidth band-pass filters FB405-10, FB490-10, FB520-10, FB590-10 (Thorlabs Inc), and ZET690/10/X (Chroma Technology Corp), using the following formula:

$$
C=\frac{I_{\mathrm{s}}-I_{\mathrm{m}}}{I_{\mathrm{s}}} \cdot 100 \%
$$

where $I_{\mathrm{s}}$ and $I_{\mathrm{m}}$ are the average intensities of the selected areas (typically $\sim 100 \mu \mathrm{m}^{2}$ ) on the substrate and 2D material, respectively.

\section{Optical contrast simulation}

The complex reflectivity coefficient was calculated using the transfer matrix method [31] and the averaged optical contrast was calculated based on the method introduced in [24], 
by integrating the reflectance over the incident angles between $0-\theta_{\max }\left(\sin \theta_{\max }=\mathrm{NA}\right)$, taking into account the appropriate angular contributions of light intensity and averaging an equal contribution from the TE and TM polarisation. All simulations considered the $50 \times$ objective $(\mathrm{NA}=0.75)$. The complex refractive indices of $\mathrm{SiO}_{2}$ and $\mathrm{Si}$ are taken from [32] and [33], and those of $\mathrm{Ti}$ and $\mathrm{Au}$ were either determined by ellipsometry or taken from [34]. The optical thickness of $d=0.34 \mathrm{~nm}$ for used for monolayer graphene [35]. Different values of the complex refractive index of graphene, $\tilde{n}=n-i \kappa$, are reported in literature, ranging from $n=2.0-3.0$ and $\kappa=0.41-2.03[16-18,36]$. We found that $\tilde{n}=2.0-1.1 i$, which was used throughout this work, provides the best fit for most experimental results on thin Au. The effect of using different complex refractive indices of graphene is demonstrated in Suppl. figure S4. There is also a variation of monolayer GO $\tilde{n}$ and $d$ in literature: $n=1.2-2.0, \kappa=0.1-0.7$, and $d=0.7$ $-1.5 \mathrm{~nm}[37-40]$, most likely caused by the variability of the oxygen-containing groups of GO. In our case, a good match between the experiments and simulations was found for $\tilde{n}=1.65-$ $0.2 i$ and $d=1.3 \mathrm{~nm}$. 


\section{Results and discussion}

First, we simulated the optical contrast spectra of monolayer graphene deposited on the heterostructure to get a guiding idea on how it depends on Au thickness in the range of $0-20$ $\mathrm{nm}$ (thicker films have only modest contrast $<2 \%$ ). Optical properties of thin Au films vary with their thickness (Suppl. figure S1). In the initial simulation, we used the experimentally determined complex refractive index of $13 \mathrm{~nm}$ Au film as a compromise between the ultrathin films $(<5 \mathrm{~nm})$ and the bulk film $(>100 \mathrm{~nm})$. The simulated contrast map in figure 2a provides a practical overview of how the optical contrast can be tuned by varying the thickness of $\mathrm{Au}$ film. A high-contrast region is found for thin Au films below $10 \mathrm{~nm}$ in thickness, with maximum positive contrast of $12.6 \%$ for $\sim 3 \mathrm{~nm}$ Au at $460 \mathrm{~nm}$ and a minimum negative contrast of $-9.3 \%$ for $\sim 7 \mathrm{~nm} \mathrm{Au}$ at $410 \mathrm{~nm}$. Thick Au films beyond $13 \mathrm{~nm}$ provide only a low optical contrast of less than $3 \%$.

To test the simulation predictions, we fabricated the heterostructures (figure 1) with a range of Au film thickness $(2.1,4.6,7.5,10.1$, and $13.0 \mathrm{~nm}$, respectively, as measured by XRR) and exfoliated graphene onto them. Au films of $2-8 \mathrm{~nm}$ in thickness were found optimal for high graphene visibility as indicated by the optical contrast data shown in figure $2 b-d$. The black contrast curves are experimentally determined from the reflectance spectra using Eq. (1), and the diamond markers are optical image contrasts determined using a range of band-pass filters and Eq. (2) (Methods). The insets are optical micrographs taken with a $520 \mathrm{~nm}$ bandpass filter with the monolayer graphene region marked (1L). The two sets of experimental contrast data show consistent spectral profiles, with slight deviations accountable within the experimental uncertainties, which are likely to be caused by a combination of several factors, such as instrumental differences between the two methods, surface contamination, and surface roughness of $\mathrm{Au}$ (see discussion of Suppl. figure S5). The reflectance-derived contrast maximum and minimum were found to be $5.0 \%$ for $2.1 \mathrm{~nm} \mathrm{Au}$ at $502 \mathrm{~nm}$ and $-8.0 \%$ for 4.6 
$\mathrm{nm} \mathrm{Au}$ at $452 \mathrm{~nm}$. In contrast, the optical image contrast of monolayer graphene without any filter (blue mark in figure $2 b-d)$ is much lower $(2.2-2.7 \%)$, since it is averaged across the whole visible spectrum.

To provide a better comparison between the experimental and simulation results, we calculated the contrast spectra using the experimentally determined optical constants of $\mathrm{Au}$ and $\mathrm{Ti}$ (different to the values used in the figure $2 \mathrm{a}$ simulation). The simulated contrast spectra in figure $2 b-d$ (red curves) match the experimental results well, especially for the contrast measured using the band-pass filters. The deviations observed between the measured and simulated contrast spectra are also caused by the uncertainties in both methods (Suppl. figure S5 discussion). The maximum experimental and simulated contrast of monolayer graphene on bulk (100 nm) Au film are 1.8\% and 1.5\%, respectively (see Suppl. figures S6 and S7). The heterostructures composed of thin Au films therefore increase the optical contrast of graphene by up to 3 times. Optical contrast data of monolayer graphene on all thin Au films $(2-13 \mathrm{~nm})$ are presented in Suppl. figure S5. Two-point resistance measurements show that only the thinnest $\mathrm{Au}$ film $(2.1 \mathrm{~nm})$ suffers from increased resistivity $\left(4.5 \times 10^{-7} \Omega \mathrm{m}\right)$ while the resistivity of $4.5-100 \mathrm{~nm} \mathrm{Au}\left(2.0-4.4 \times 10^{-8} \Omega \mathrm{m}\right)$ is similar to the literature bulk value [41]. 

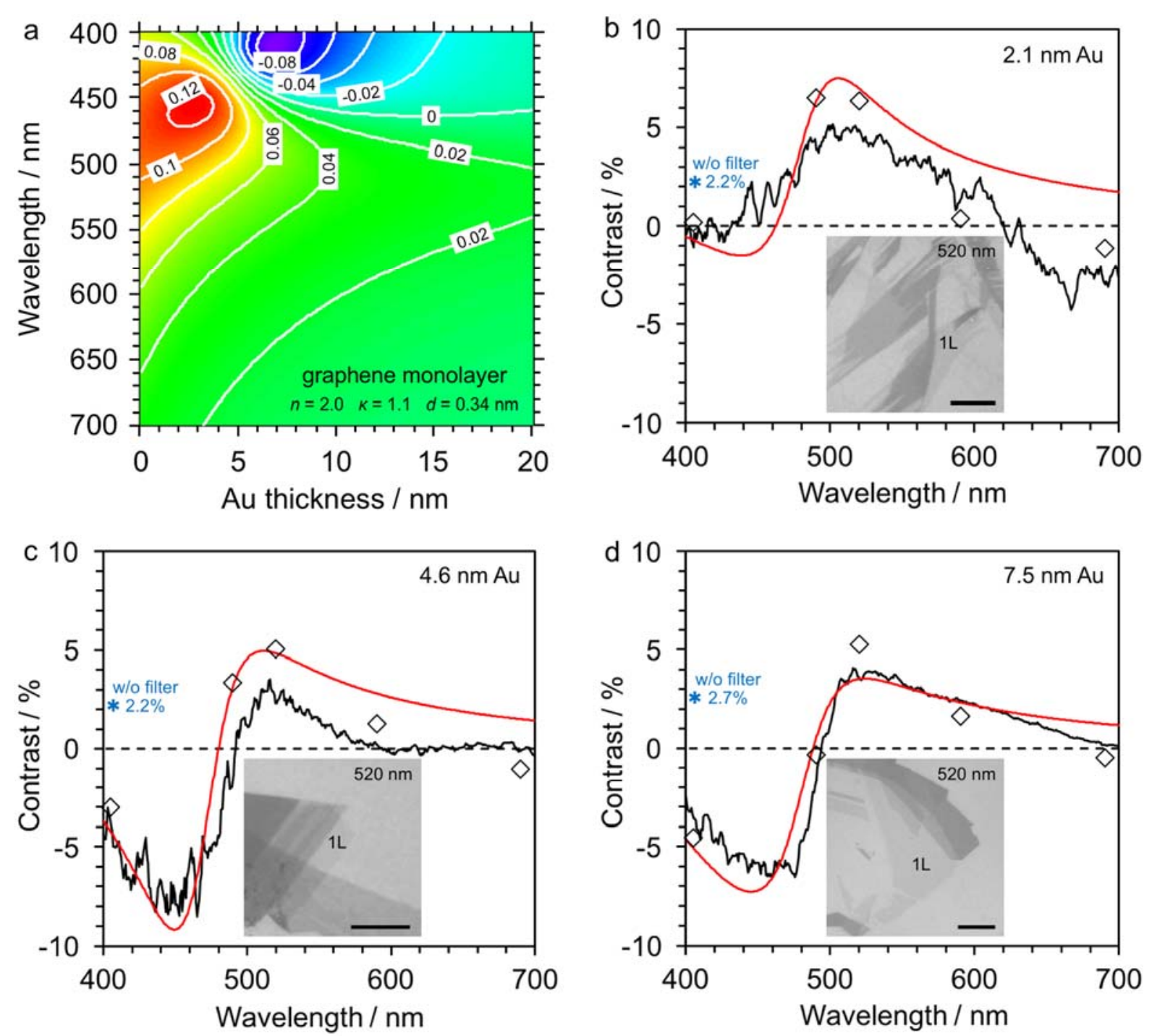

Figure 2 | Optical contrast of graphene on thin Au films. a, Simulated contrast map of monolayer graphene as a function of Au thickness and wavelength on $1 \mathrm{~nm} \mathrm{Ti} / 90 \mathrm{~nm} \mathrm{SiO} / / \mathrm{Si}$ substrate, using literature refractive indices for bulk $\mathrm{Ti}, \mathrm{SiO}_{2}$, and $\mathrm{Si}$, and experimentally determined ones for $\mathrm{Au}(13 \mathrm{~nm})$. The contours are annotated with their fractional positive/negative contrast values (e.g., 0.02 indicates a positive contrast of $2 \%$ ). b-d, Optical contrast of monolayer graphene on 2.1, 4.6, and $7.5 \mathrm{~nm} \mathrm{Au}$ films on $1 \mathrm{~nm} \mathrm{Ti} / 93 \mathrm{~nm} \mathrm{SiO} / 2 / \mathrm{Si}$ substrate (taken using 50× objective). The black curves are contrasts determined from the reflectance spectra using Eq. (1). The diamonds are contrasts determined using Eq. (2) from the optical images using band-pass filters $(405,490,520,590$, and $690 \mathrm{~nm}$, all $10 \mathrm{~nm}$ bandwidth). The red curves are simulations based on the experimentally determined optical constants and thicknesses of $\mathrm{Au}$ and Ti. The blue star symbols correspond to the optical image contrast without a filter. Error bars are not shown as they are comparable in size to the markers $(0.0-0.7 \%)$. The insets show greyscale optical images of the graphene layers (using $520 \mathrm{~nm}$ band-pass filter). Optical constants of $n=2.0$ and $\kappa=1.1$ and thickness $d=0.34 \mathrm{~nm}$ were used for graphene in all simulations. All scale bars correspond to $15 \mu \mathrm{m}$. 
In figure $3 \mathrm{a}$, we further examine the optical contrast of graphene on $7.5 \mathrm{~nm}$ Au taken without and with different band-pass optical filters (10 $\mathrm{nm}$ bandwidth). The images were converted to greyscale and their substrate intensities adjusted to a similar level for ease of comparison. It is evident that the $520 \mathrm{~nm}$ filter (the original optical image is in figure $3 \mathrm{~b}$ ) provides the best visibility and distinctly reveals graphene layers of different thickness, in accord with the results in figure $2 \mathrm{~d}$. Unlike the minimum negative contrast at $\sim 450 \mathrm{~nm}$, the positive contrast maximum at $520 \mathrm{~nm}$ allows direct inspection of graphene under an optical microscope due to the preferential sensitivity of the human eye to green wavelengths. Processed and unprocessed optical images are shown in Suppl. figure S8.

The measured optical contrast spectra of few-layer graphene $(1-5 \mathrm{~L})$ are shown in figure 3c. Here we used the $100 \times$ objective with a smaller spot-size to mitigate the uncertainties due to small crystals size and sample drifting. The measured spectra demonstrate that: 1) the whole spectrum slightly redshifts with increasing number of layers, 2) the absolute maximum/minimum values of the contrast increase linearly with the number of layers (inset of figure 3c) to the first approximation, consistent with previous reports on $\mathrm{SiO}_{2} / \mathrm{Si}$ substrates [17]. The layer identification was further confirmed by Raman spectroscopy (figure 3d) and AFM (figure 3e). The symmetric $2 \mathrm{D}$ mode $\left(\sim 2650 \mathrm{~cm}^{-1}\right)$ in the Raman spectra, redshifted in respect to few-layer graphene and stronger in intensity than the $\mathrm{G}$ mode $\left(\sim 1580 \mathrm{~cm}^{-1}\right)$, is a unique fingerprint of monolayer graphene. The intensity of the G mode linearly increases and the $2 \mathrm{D}$ mode broadens and blueshifts with the increasing number of layers, a behaviour characteristic for few-layer graphene [42, 43]. No D mode $\left(\sim 1360 \mathrm{~cm}^{-1}\right)$ was detected, indicating high crystal quality. Figure 3 e shows the AFM topography image of an area marked in figure $3 \mathrm{~b}$. Although the scan was challenging due to variation in surface roughness, it produced an average step height of $\sim 0.34 \pm 0.10 \mathrm{~nm}$ between monolayer graphene and the substrate (figure 3e inset), expected for monolayer graphene [44]. 

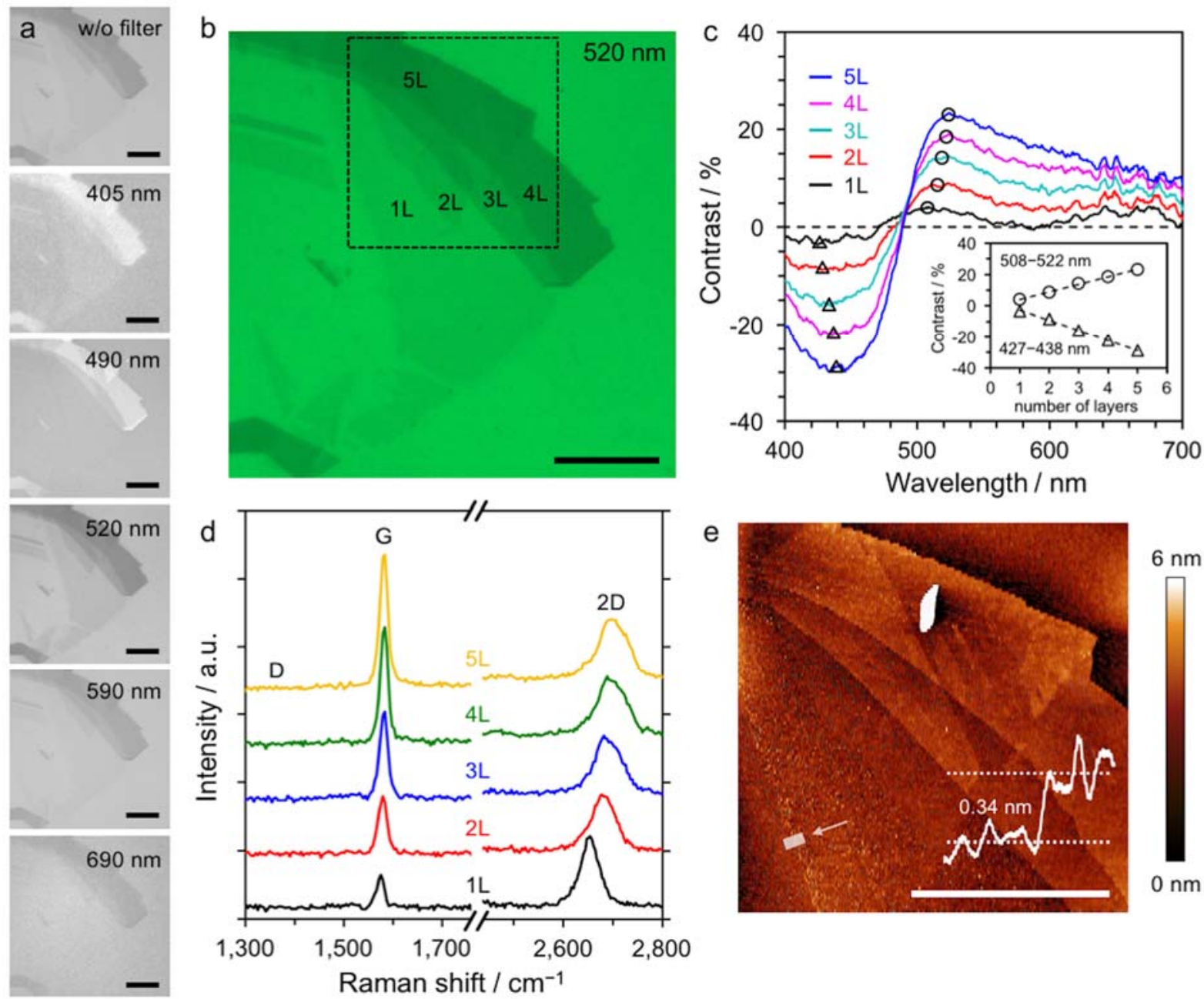

Figure 3 Characterisation of graphene layers on $7.5 \mathbf{~ n m ~ A u ~ f i l m . ~ a , ~ S e r i e s ~ o f ~ o p t i c a l ~}$ images of graphene on $7.5 \mathrm{~nm} \mathrm{Au} / 1 \mathrm{~nm} \mathrm{Ti} / 93 \mathrm{~nm} \mathrm{SiO} / / \mathrm{Si}$ substrate, taken without and with a range of band-pass filters $(405,490,520,590,690 \mathrm{~nm}) . \mathbf{b}$, Optical image taken using a $520 \mathrm{~nm}$ filter, with the number of graphene layers indicated. c, Optical contrast spectra of the graphene layers (1-5L) in b taken using a 100× objective. Inset: positive contrast maxima and negative contrast minima as a function of the number of layers. $\mathbf{d}$, Raman spectra of the graphene layers in $\mathbf{b}$ with the main vibration modes, $\mathrm{G}$ and $2 \mathrm{D}$, annotated (spectra are offset for clarity). e, topographic AFM image of a portion of the sample, highlighted in $\mathbf{b}$ by a black dashed rectangle. Inset: height profile of a monolayer-substrate step, highlighted in e by a white rectangle. All scale bars correspond to $15 \mu \mathrm{m}$. 
GO is related to graphene since it is obtained by surface oxidation of graphene or graphite. The extent and nature of the oxygen-containing functional groups in GO varies widely and is dependent on the preparation method [45, 46]. GO applications include sensors, composites, and ink-based technologies $[47,48]$. Similarly to graphene, GO is also highly transparent in the visible range, with the experimentally measured optical contrast of $3.6 \%$ on $300 \mathrm{~nm} \mathrm{SiO} / / \mathrm{Si}$ using a $100 \times$ objective [38]. Applying the same methodology as for graphene, we found that the stratified heterostructures can also significantly enhance the optical visibility of GO, across a wide range of Au thicknesses. The simulation in figure 4a shows that contrast well above 4\% can be achieved for $0-20 \mathrm{~nm}$ Au films, with the highest contrast found for $2-$ $10 \mathrm{~nm} \mathrm{Au}$. The simulation reveals a maximum positive contrast of $14.8 \%$ for $5.0 \mathrm{~nm}$ Au at 465 $\mathrm{nm}$ and a minimum negative contrast of $-14.7 \%$ for $5.5 \mathrm{~nm} \mathrm{Au}$ at $405 \mathrm{~nm}$. The experimental optical contrast data for monolayer GO on $2.1-7.5 \mathrm{~nm}$ Au films are shown in figure 4b-d. The black curves are the optical contrast spectra determined from the reflectance measurements, the diamond markers are the optical contrast data determined from the optical images using band-pass filters, and the red curves are the contrast spectra simulated using the experimentally determined optical constants and thicknesses of $\mathrm{Au}$ and $\mathrm{Ti}$ layers. The experimental and simulated data are in good agreement. The reflectance contrast maximum and minimum were found experimentally to be $16.6 \%$ for $4.6 \mathrm{~nm}$ Au at $517 \mathrm{~nm}$ and $-15.2 \%$ for $4.6 \mathrm{~nm} \mathrm{Au}$ at $440 \mathrm{~nm}$. In comparison, the maximum optical contrast of monolayer GO on bulk $\mathrm{Au}$ is only $3.3 \%$ (Suppl. figure S7). The stratified heterostructure therefore remarkably improves the optical contrast of a monolayer GO on Au film by up to 5 times, in comparison to bulk $\mathrm{Au}$ substrate, which is also 2 times higher than on the bare $93 \mathrm{~nm} \mathrm{SiO}_{2} / \mathrm{Si}$ substrate. Optical contrast data of monolayer GO on all Au films $(2-13 \mathrm{~nm})$ are found in Suppl. figure S9. 
Having identified the optimal Au thickness for the highest GO optical contrast, we further characterised the optical visibility of multilayer GO. Figure 5a shows the optical images of mono- and multi-layer GO flakes drop-cast on a $4.6 \mathrm{~nm} \mathrm{Au}$ film, taken with different bandpass filters. The images are processed the same way as in figure $3 \mathrm{a}$ for ease of comparison. The $520 \mathrm{~nm}$ filter provides the best visibility of the GO layers (figure 5a-b), which is consistent with the measured contrast spectrum and simulations (figure 4c). The measured optical contrast spectra of the GO layers (1-4L), shown in figure 5c, behaves similarly to that of graphene. The whole spectrum slightly redshifts and the absolute maximum/minimum values of the contrast increase linearly with the number of layers (inset of figure $5 \mathrm{c}$ ). The Raman spectra of the GO layers are shown in figure $5 \mathrm{~d}$. Unlike for graphene, there is no distinct 2D mode in the Raman spectrum, instead, high-intensity D and G modes are present. The positions of the D and G modes are not sensitive to the GO thickness but their intensities increase linearly with the number of layers, which is consistent with previous studies $[38,39]$. Figure 5e shows the AFM phase image of a monolayer region marked in figure $5 \mathrm{~b}$. The monolayer-substrate step-height extracted from the AFM topography mode was $\sim 0.99 \pm 0.30 \mathrm{~nm}$ (inset of figure $5 \mathrm{e}$ ), which is well within the scope of reported monolayer GO thicknesses [38, 40]. 

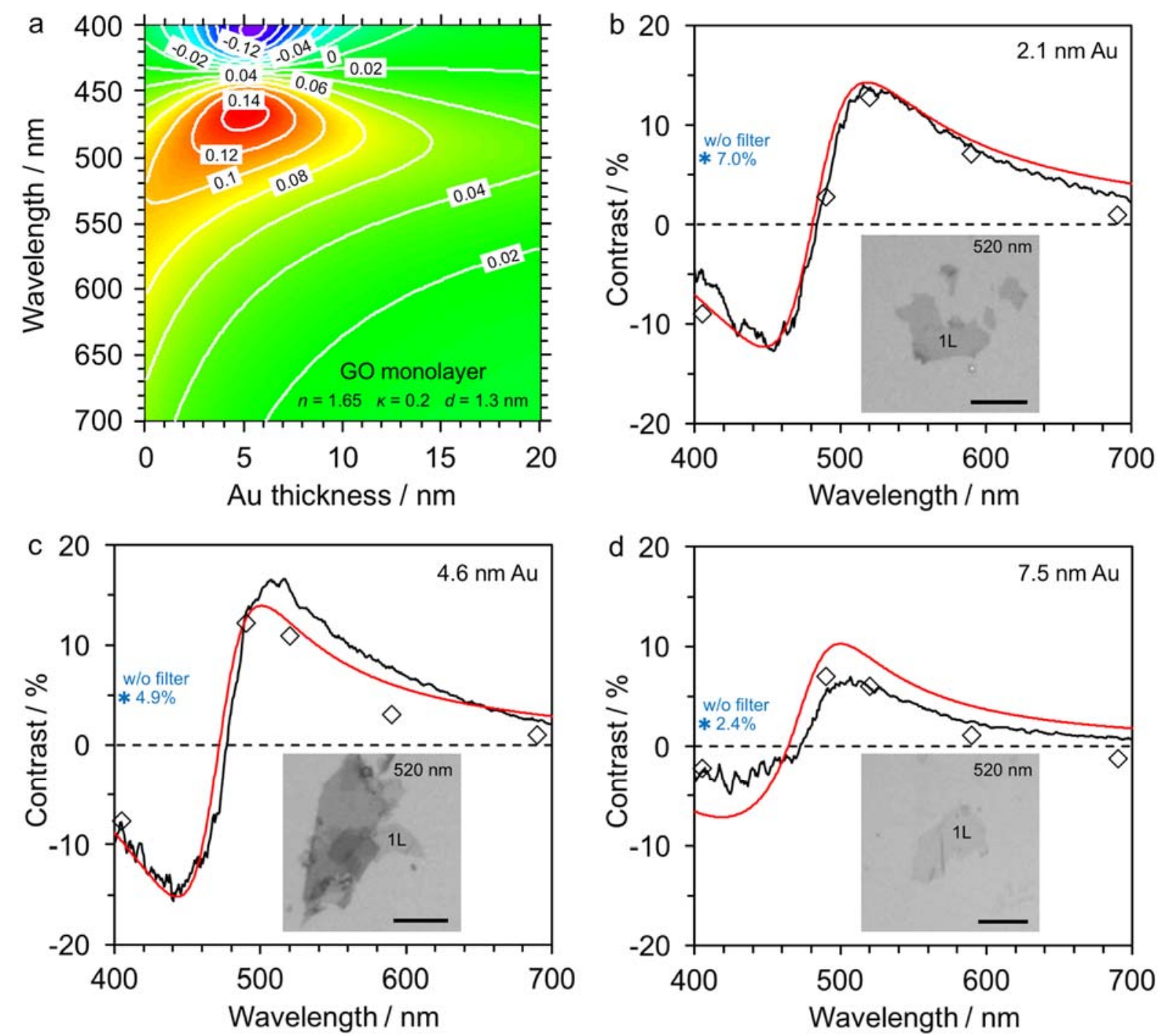

Figure 4 | Optical contrast of GO on thin Au films. a, Simulated contrast map of monolayer $\mathrm{GO}$ as a function of $\mathrm{Au}$ thickness and wavelength on $1 \mathrm{~nm} \mathrm{Ti} / 90 \mathrm{~nm} \mathrm{SiO} / 2 / \mathrm{Si}$ substrate, using library refractive indices for bulk $\mathrm{Ti}, \mathrm{SiO}_{2}$, and $\mathrm{Si}$, and experimental for $\mathrm{Au}(13 \mathrm{~nm} \mathrm{Au})$. The contours are annotated with their fractional positive/negative contrast values. b-d, Optical contrast of monolayer GO drop-cast on 2.1, 4.6, and $7.5 \mathrm{~nm} \mathrm{Au}$ films on $1 \mathrm{~nm} \mathrm{Ti} / 93 \mathrm{~nm} \mathrm{SiO} / \mathrm{Si}$ substrate (taken using 50× objective). The data presentation adheres to the same scheme as in figure 2. Error bars are not shown as they are comparable in size to the markers $(0.1-1.3 \%)$. The insets show greyscale optical images of the GO layers (taken with a $520 \mathrm{~nm}$ band-pass filter). Optical constants of $n=1.65$ and $\kappa=0.2$ and thickness $d=1.3 \mathrm{~nm}$ were used for GO in all simulations. All scale bars correspond to $15 \mu \mathrm{m}$. 


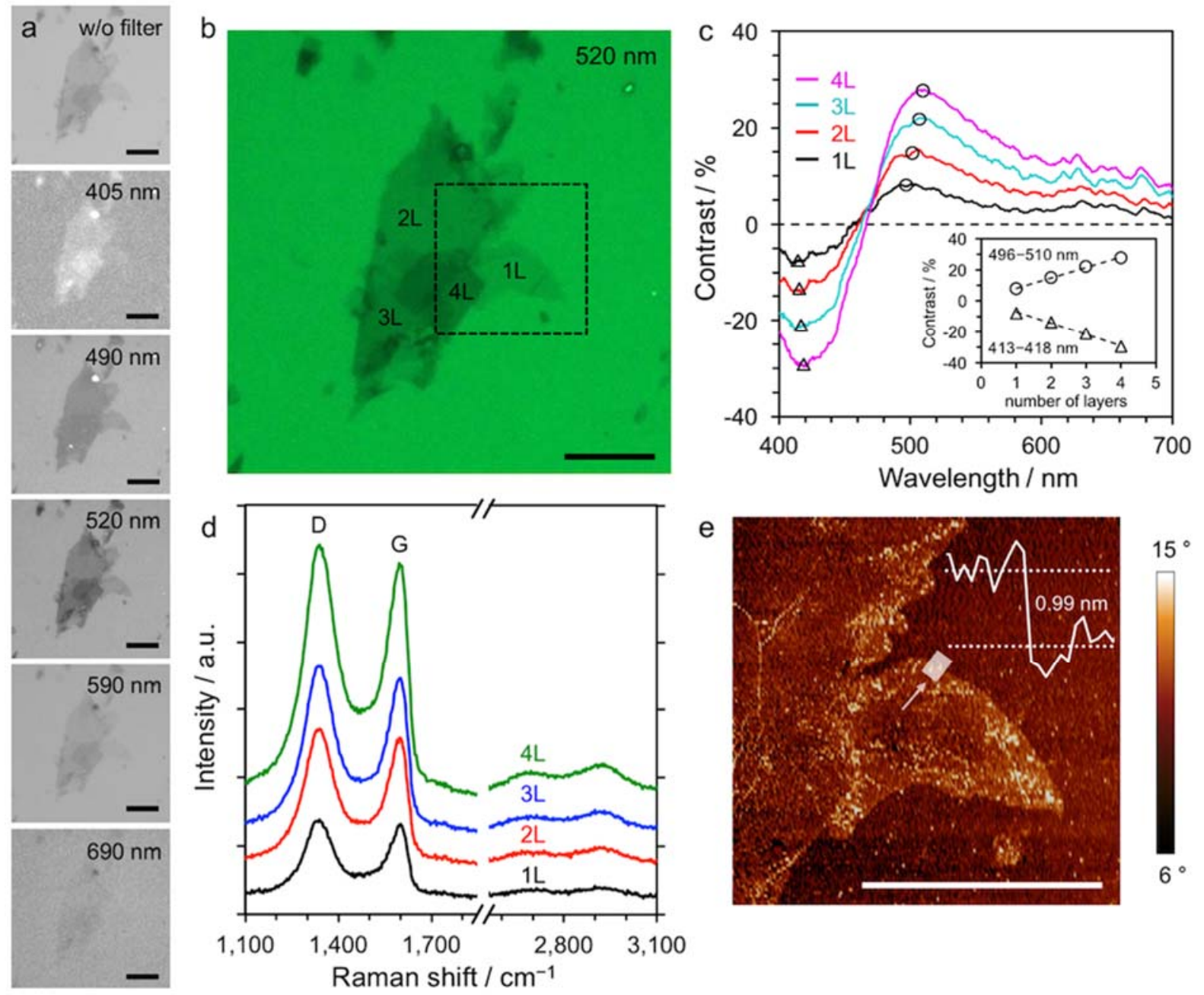

Figure 5 | Characterisation of GO layers on $4.6 \mathrm{~nm}$ Au film. a, Series of optical images of GO on $4.6 \mathrm{~nm} \mathrm{Au} / 1 \mathrm{~nm} \mathrm{Ti} / 93 \mathrm{~nm} \mathrm{SiO} / \mathrm{Si}$ substrate, taken without and with a range of bandpass filters $(405,490,520,590,690 \mathrm{~nm})$. b. Optical image using a $520 \mathrm{~nm}$ band-pass filter, with the number of GO layers indicated. c, Optical contrast spectra of GO layers (1-4L) in b taken using a $100 \times$ objective. Inset: positive contrast maxima and negative contrast minima as a function of the number of layers. d, Raman spectra of the GO layers in $\mathbf{b}$ with the main vibration modes, D and G, annotated (spectra are offset for clarity). e, phase AFM image of a portion of the sample, highlighted in $\mathbf{b}$ by a black dashed rectangle. Inset: height profile of a monolayer-substrate step, highlighted in e by a white rectangle. All scale bars correspond to 10 $\mu \mathrm{m}$. 
We now proceed to summarise the results in figure 6 , which shows the positive maximum contrast for both 2D materials obtained from the reflectance spectra (full markers) and simulations (empty markers) as a function of the $\mathrm{Au}$ film thickness. Contrast on bulk $\mathrm{Au}$ film $(100 \mathrm{~nm})$ and bare $93 \mathrm{~nm} \mathrm{SiO}_{2} / \mathrm{Si}$ substrate $(0 \mathrm{~nm})$ are also plotted for comparison. It is clear that the thin Au films $(2-8 \mathrm{~nm})$ provide significant improvement in the optical contrast for both 2D materials over bulk $\mathrm{Au}$, up to about 3 times and 5 times for monolayer graphene and GO, respectively, based on experimental results (or 5 and 6 times, based on simulations). Moreover, the optical contrast of monolayer GO on thin Au films increases up to about 2 times in comparison to the bare $93 \mathrm{~nm} \mathrm{SiO} / / \mathrm{Si}$ substrate. Positive contrast maxima exist for monolayers of both studied 2D materials in the range of $500-530 \mathrm{~nm}$. The use of blue/green band-pass filters in this region increases the visibility of 2D materials on these films by ca. 2 5 times over the case without a filter. The negative contrast minima in the range of $400-440$ $\mathrm{nm}$ can be exploited by the use of violet/deep-blue filters, which is however less practical due to the poor sensitivity of the human eye and most cameras at these wavelengths. The contrast data of figure 6 are summarized in Suppl. table S1. 


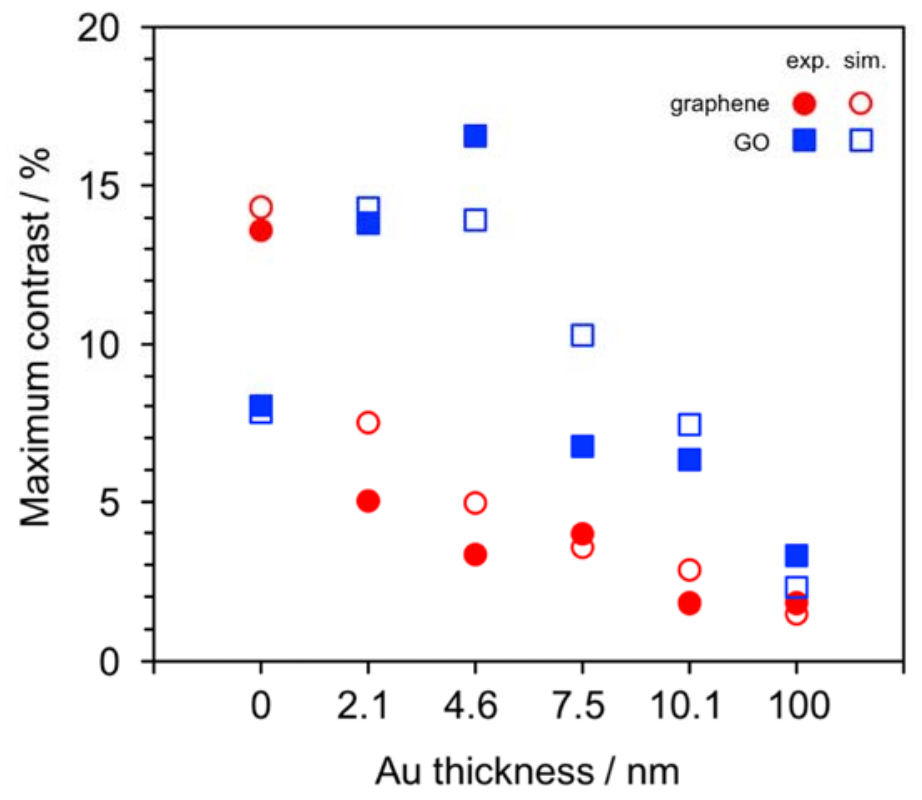

Figure 6 |Summary of the optical contrast of graphene and GO. Comparison of the maximum experimental reflectance contrast (full markers) and simulated contrast (empty markers) of graphene (red circles) and GO (blue squares) as a function of Au thickness, including the simulation for the bare $93 \mathrm{~nm} \mathrm{SiO} / / \mathrm{Si}$ substrate $(0 \mathrm{~nm} \mathrm{Au})$. The average standard deviations are estimated to be $\pm(0.15-0.37) \%$.

The enhanced optical contrast of ultrathin 2D materials such as graphene and GO originates from the modified complex reflectivity of the underlying heterostructure substrate [24]. In general, the contrast increases with decreasing reflectance of the substrate, however, the phase of the reflection coefficient is also essential. Furthermore, the contrast critically depends on the conditions of light incidence, including the wavelength, incident angle and polarisation. To illustrate this, we simulated contrast maps for monolayer graphene and GO (figure 7) at several different conditions specified by polarisation and incidence angle $(\theta)$, namely: TE, $\theta=0^{\circ}$ (left column); TE, $\theta=45^{\circ}$ (middle column), and TM, $\theta=45^{\circ}$ (right column). The vertical logarithmic axis denotes the absolute value of the complex reflection coefficient ( $|r|$, where reflectance $R=|r|^{2}$ ) of the bare substrate without a 2D material. The horizontal axis is the phase of the complex reflection coefficient of the bare substrate. All the contrast maps are calculated at $500 \mathrm{~nm}$ wavelength using the same refractive indices and thicknesses of 
graphene and GO as in the previous simulations. Figure 7 reveals several general characteristics about the contrast of graphene and GO, which also apply to other 2D materials: 1) The contrast increases with diminishing reflectivity of the substrate and so a high contrast substrate should normally have a low reflectivity. 2) Even on a substrate of a very low reflectivity, a phase always exists for which the contrast is zero. 3) For every 2D material, specific phase conditions are found for the contrast maximum and minimum, dependent on the polarisation and wavelength of light. For graphene, the contrast maxima phases are about $32^{\circ}$ and $153^{\circ}$ for TE and TM polarisation, respectively. For GO, the contrast maxima phases are about $71^{\circ}$ and $116^{\circ}$ for TE and TM polarisation, respectively. The phases of the contrast minima are shifted by $\left.180^{\circ} .4\right)$ The complex reflectivity and hence the contrast are dependent on the incident angle of light. Therefore, when a high magnification objective with large NA is used, the overall contrast has to be averaged over all incident angles.

Every substrate can be marked as a point on the contrast map, based on its complex reflectivity coefficient, as shown in figure 7. Bulk Au (red diamond) has a high reflectivity, so it only yields a modest optical contrast. Such a feature is shared by all reflective metals, such as $\mathrm{Ag}, \mathrm{Cu}$ and $\mathrm{Al}$. The reflectivity of thin $\mathrm{Au}$ film structures is lower than that of bulk Au and closer to the optimal phase conditions in most of the cases, therefore enhancing the optical contrast for graphene and GO. If light were solely incident according to the optimal conditions, a very high contrast exceeding $20 \%$ for both monolayer graphene and GO could be achieved on various substrates. The details of the contrast data on these substrates are presented in Suppl. table S2. 


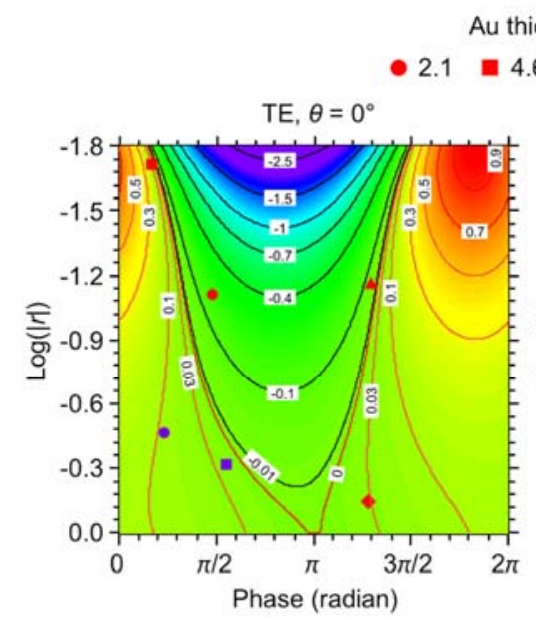

$\begin{array}{lc}\text { Au thickness } / \mathrm{nm} & \mathrm{SiO}_{2} \text { thickness } / \mathrm{nm} \\ 4.6-7.5-100 & -90=290\end{array}$
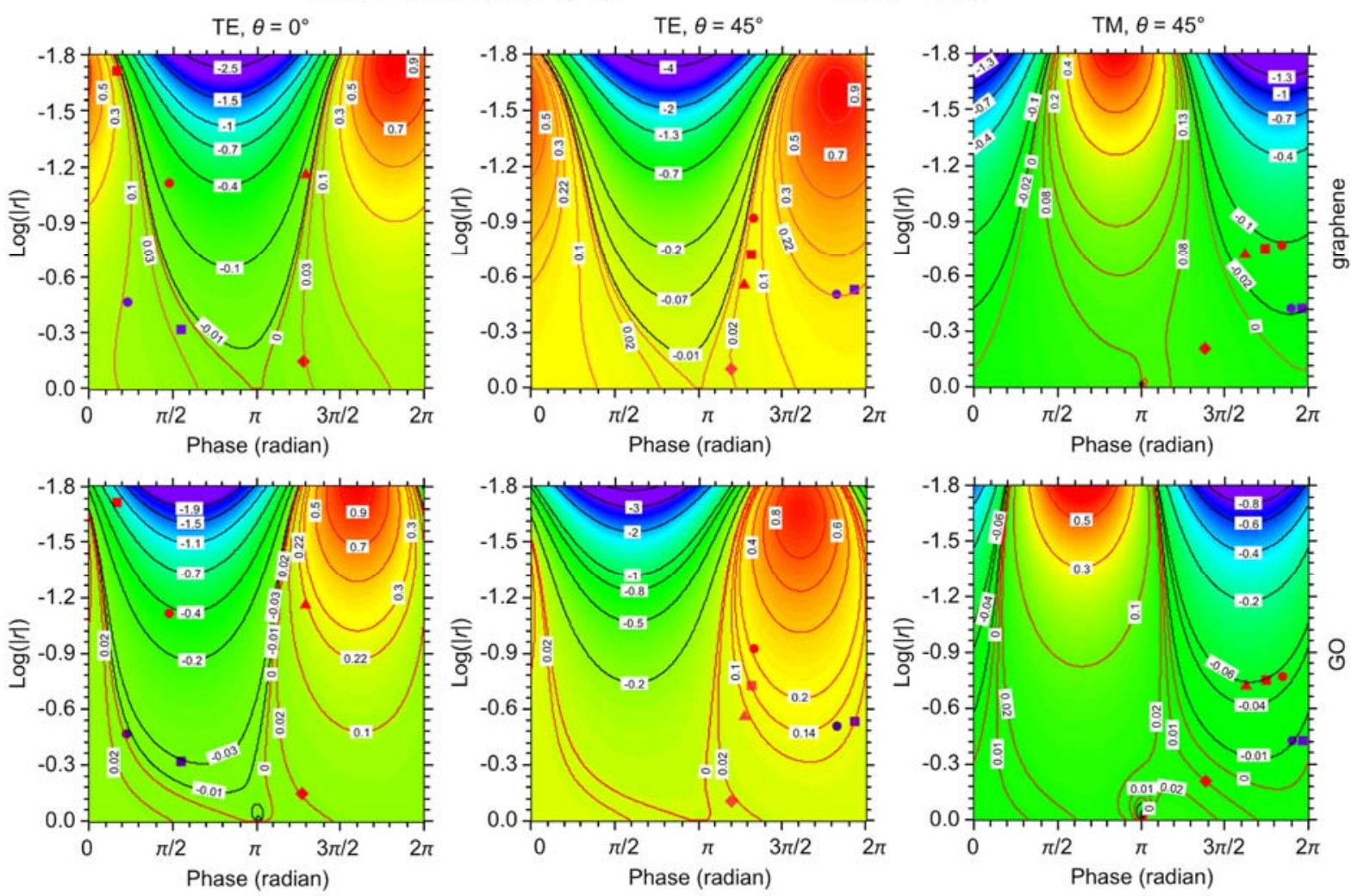

Figure 7 | Simulated contrast maps under various incident conditions. Simulations for monolayer graphene (upper panels) and monolayer GO (lower panels). Left column: TE polarisation, normal incidence. Middle column: TE polarisation, $45^{\circ}$ incidence. Right column: TM polarisation, $45^{\circ}$ incidence. All the contrast maps are calculated at $500 \mathrm{~nm}$ wavelength. The contours are annotated with their fractional positive/negative contrast values. The different substrates used in this work are highlighted in the maps by different markers. The same refractive indices and 2D material thicknesses as in the previous simulations were used.

\section{Conclusion}

In summary, we demonstrate that the stratified heterostructures composed of $2-8 \mathrm{~nm}$ Au films on $1 \mathrm{~nm} \mathrm{Ti} / 93 \mathrm{~nm} \mathrm{SiO} / \mathrm{Si}$ substrate can significantly enhance the optical visibility of graphene and GO. In comparison to bulk $\mathrm{Au}$, the experimentally determined optical contrast of monolayer graphene and GO on these thin Au films is enhanced by up to 3 and 5 times, respectively. Furthermore, thin $4.6 \mathrm{~nm}$ Au improves the optical visibility of GO up to 2 times over the bare $93 \mathrm{~nm} \mathrm{SiO}_{2} / \mathrm{Si}$ substrate. Only the thinnest $\mathrm{Au}$ film $(2.1 \mathrm{~nm})$ suffers from 
increased resistivity, which should be taken into account in the relevant device designs. The experimental results are in good agreement with theoretical simulations, which take into account the effects of different incident angles and polarisations. This concept is demonstrated for $\mathrm{Au}$, but the same methodology can be adopted for other metals. These results provide a clear pathway for those who wish to utilise 2D materials on metallic films in research and applications including, but not limited to, plasmonics, photonics, catalysis and sensors.

\section{Acknowledgements}

This work was funded by the EPSRC (grant No. EP/N025938/1).

\section{References}

[1] M. Buscema, G.A. Steele, H.S.J. van der Zant, A. Castellanos-Gomez, The effect of the substrate on the Raman and photoluminescence emission of single-layer $\mathrm{MoS}_{2}$, Nano Res. 7 (2014) 1-11.

[2] J. Hui, X. Zhou, R. Bhargava, A. Chinderle, J. Zhang, J. Rodríguez-López, Kinetic Modulation of Outer-Sphere Electron Transfer Reactions on Graphene Electrode with a Subsurface Metal Substrate, Electrochim. Acta 211 (2016) 1016-1023.

[3] Q.H. Wang, Z. Jin, K.K. Kim, A.J. Hilmer, G.L.C. Paulus, C.J. Shih, M.H. Ham, J.D. Sanchez-Yamagishi, K. Watanabe, T. Taniguchi, J. Kong, P. Jarillo-Herrero, M.S. Strano, Understanding and controlling the substrate effect on graphene electron-transfer chemistry via reactivity imprint lithography, Nat. Chem. 4 (2012) 724-732.

[4] L. Wang, I. Meric, P.Y. Huang, Q. Gao, Y. Gao, H. Tran, T. Taniguchi, K. Watanabe, L.M. Campos, D.A. Muller, J. Guo, P. Kim, J. Hone, K.L. Shepard, C.R. Dean, One-

Dimensional Electrical Contact to a Two-Dimensional Material, Science 342 (2013) 614-617. [5] K. Uosaki, G. Elumalai, H. Noguchi, T. Masuda, A. Lyalin, A. Nakayama, T. Taketsugu, Boron Nitride Nanosheet on Gold as an Electrocatalyst for Oxygen Reduction Reaction: Theoretical Suggestion and Experimental Proof, J. Am. Chem. Soc. 136 (2014) 6542-6545. [6] J. Mertens, A.L. Eiden, D.O. Sigle, F. Huang, A. Lombardo, Z. Sun, R.S. Sundaram, A. Colli, C. Tserkezis, J. Aizpurua, S. Milana, A.C. Ferrari, J.J. Baumberg, Controlling subnanometer gaps in plasmonic dimers using graphene, Nano Lett. 13 (2013) 5033-5038. [7] K. Choi, Y.T. Lee, S. Im, Two-dimensional van der Waals nanosheet devices for future electronics and photonics, Nano Today 11 (2016) 626-643.

[8] M. Parzefall, P. Bharadwaj, A. Jain, T. Taniguchi, K. Watanabe, L. Novotny, Antennacoupled photon emission from hexagonal boron nitride tunnel junctions, Nat. Nanotechnol. 10 (2015) 1058-1063. 
[9] M.-E. Kleemann, R. Chikkaraddy, E.M. Alexeev, D. Kos, C. Carnegie, W. Deacon, A.C. de Pury, C. Große, B. de Nijs, J. Mertens, A.I. Tartakovskii, J.J. Baumberg, Strong-coupling of $\mathrm{WSe}_{2}$ in ultra-compact plasmonic nanocavities at room temperature, Nat. Commun. 8 (2017) 1296.

[10] S. Bae, H. Kim, Y. Lee, X. Xu, J.S. Park, Y. Zheng, J. Balakrishnan, T. Lei, H. Ri Kim, Y.I. Song, Y.J. Kim, K.S. Kim, B. Özyilmaz, J.H. Ahn, B.H. Hong, S. Iijima, Roll-to-roll production of 30-inch graphene films for transparent electrodes, Nat. Nanotechnol. 5 (2010) 574-578.

[11] K.S. Kim, Y. Zhao, H. Jang, S.Y. Lee, J.M. Kim, K.S. Kim, J.-H. Ahn, P. Kim, J.-Y. Choi, B.H. Hong, Large-scale pattern growth of graphene films for stretchable transparent electrodes, Nature 457 (2009) 706-710.

[12] Y. Gao, Z. Liu, D.-M. Sun, L. Huang, L.-P. Ma, L.-C. Yin, T. Ma, Z. Zhang, X.-L. Ma, L.-M. Peng, H.-M. Cheng, W. Ren, Large-area synthesis of high-quality and uniform monolayer $\mathrm{WS}_{2}$ on reusable Au foils, Nat. Commun. 6 (2015) 8569.

[13] G. Giovannetti, P.A. Khomyakov, G. Brocks, V.M. Karpan, J. van den Brink, P.J. Kelly, Doping Graphene with Metal Contacts, Phys. Rev. Lett. 101 (2008) 026803.

[14] J. Wintterlin, M.L. Bocquet, Graphene on metal surfaces, Surf. Sci. 603 (2009) 18411852.

[15] J.M. Katzen, M. Velický, Y. Huang, S. Drakeley, W. Hendren, R.M. Bowman, Q. Cai, Y. Chen, L.H. Li, F. Huang, Hunting micrometer-sized graphene flakes on gold substrate, arXiv preprint, arXiv:1801.05622 (2018).

[16] M.R. Müller, A. Gumprich, E. Ecik, K.T. Kallis, F. Winkler, B. Kardynal, I. Petrov, U. Kunze, J. Knoch, Visibility of two-dimensional layered materials on various substrates, J. Appl. Phys. 118 (2015) 145305.

[17] Z.H. Ni, H.M. Wang, J. Kasim, H.M. Fan, T. Yu, Y.H. Wu, Y.P. Feng, Z.X. Shen, Graphene thickness determination using reflection and contrast spectroscopy, Nano Lett. 7 (2007) 2758-2763.

[18] P. Blake, E.W. Hill, A.H. Castro Neto, K.S. Novoselov, D. Jiang, R. Yang, T.J. Booth, A.K. Geim, Making graphene visible, Appl. Phys. Lett. 91 (2007) 063124.

[19] I. Jung, M. Pelton, R. Piner, D.A. Dikin, S. Stankovich, S. Watcharotone, M. Hausner, R.S. Ruoff, Simple Approach for High-Contrast Optical Imaging and Characterization of Graphene-Based Sheets, Nano Lett. 7 (2007) 3569-3575.

[20] T. Kaplas, A. Zolotukhin, Y. Svirko, Thickness determination of graphene on metal substrate by reflection spectroscopy, Opt. Express 19 (2011) 17226-17231.

[21] I. Wlasny, P. Dabrowski, Z. Klusek, Optical contrast of single-and multi-layer graphene deposited on a gold substrate, arXiv preprint, arXiv:1102.4953 (2011).

[22] G. Teo, H. Wang, Y. Wu, Z. Guo, J. Zhang, Z. Ni, Z. Shen, Visibility study of graphene multilayer structures, J. Appl. Phys. 103 (2008) 124302.

[23] S. McDonnell, R. Addou, C. Buie, R.M. Wallace, C.L. Hinkle, Defect-dominated doping and contact resistance in $\mathrm{MoS}_{2}$, ACS Nano 8 (2014) 2880-2888.

[24] X. Wang, M. Zhao, D.D. Nolte, Optical contrast and clarity of graphene on an arbitrary substrate, Appl. Phys. Lett. 95 (2009) 081102.

[25] S.H. Jang, I. Jung, Visibility of few-layer graphene oxide under modified light using bandpass filters, Journal of the Optical Society of America A 33 (2016) 2099-2107.

[26] M. Velický, M.A. Bissett, P.S. Toth, H.V. Patten, S.D. Worrall, A.N.J. Rodgers, E.W.

Hill, I.A. Kinloch, K.S. Novoselov, T. Georgiou, L. Britnell, R.A.W. Dryfe, Electron transfer kinetics on natural crystals of $\mathrm{MoS}_{2}$ and graphite, Phys. Chem. Chem. Phys. 17 (2015) 17844-17853.

[27] M. Velický, P.S. Toth, A.M. Rakowski, A.P. Rooney, A. Kozikov, C.R. Woods, A. Mishchenko, L. Fumagalli, J. Yin, V. Zólyomi, T. Georgiou, S.J. Haigh, K.S. Novoselov, 
R.A.W. Dryfe, Exfoliation of natural van der Waals heterostructures to a single unit cell thickness, Nat. Commun. 8 (2017) 14410.

[28] K.S. Novoselov, A.K. Geim, S.V. Morozov, D. Jiang, Y. Zhang, S.V. Dubonos, I.V. Grigorieva, A.A. Firsov, Electric field in atomically thin carbon films, Science 306 (2004) 666-669.

[29] Y.C. Lin, C.C. Lu, C.H. Yeh, C. Jin, K. Suenaga, P.W. Chiu, Graphene annealing: How clean can it be?, Nano Lett. 12 (2012) 414-419.

[30] K.S. Novoselov, A.H. Castro Neto, Two-dimensional crystals-based heterostructures: materials with tailored properties, Phys. Scr. 2012 (2012) 014006.

[31] M. Born, E. Wolf, Principles of Optics: Electromagnetic Theory of Propagation, Interference and Diffraction of Light, 7 ed., Cambridge University Press, Cambridge, 1999. [32] I.H. Malitson, Interspecimen Comparison of the Refractive Index of Fused Silica, J. Opt. Soc. Am. 55 (1965) 1205-1209.

[33] C.M. Herzinger, B. Johs, W.A. McGahan, J.A. Woollam, W. Paulson, Ellipsometric determination of optical constants for silicon and thermally grown silicon dioxide via a multisample, multi-wavelength, multi-angle investigation, J. Appl. Phys. 83 (1998) 3323-3336. [34] A.D. Rakić, A.B. Djurišić, J.M. Elazar, M.L. Majewski, Optical properties of metallic films for vertical-cavity optoelectronic devices, Appl. Opt. 37 (1998) 5271-5283.

[35] P.R. Wallace, The band theory of graphite, Phys. Rev. 71 (1947) 622-634.

[36] S. Cheon, K.D. Kihm, H.g. Kim, G. Lim, J.S. Park, J.S. Lee, How to Reliably Determine the Complex Refractive Index (RI) of Graphene by Using Two Independent Measurement Constraints, Sci. Rep. 4 (2014) 6364.

[37] I. Jung, M. Vaupel, M. Pelton, R. Piner, D.A. Dikin, S. Stankovich, J. An, R.S. Ruoff, Characterization of Thermally Reduced Graphene Oxide by Imaging Ellipsometry, The Journal of Physical Chemistry C 112 (2008) 8499-8506.

[38] H. Yang, H. Hu, Y. Wang, T. Yu, Rapid and non-destructive identification of graphene oxide thickness using white light contrast spectroscopy, Carbon 52 (2013) 528-534.

[39] D. Kostiuk, M. Bodik, P. Siffalovic, M. Jergel, Y. Halahovets, M. Hodas, M. Pelletta, M. Pelach, M. Hulman, Z. Spitalsky, M. Omastova, E. Majkova, Reliable determination of the few-layer graphene oxide thickness using Raman spectroscopy, J. Raman Spectrosc. 47 (2016) 391-394.

[40] J. Zhang, H. Yang, G. Shen, P. Cheng, J. Zhang, S. Guo, Reduction of graphene oxide vial-ascorbic acid, Chem. Commun. (Cambridge, U. K.) 46 (2010) 1112-1114.

[41] D.R. Lide, Handbook of Chemistry and Physics, 74th ed., CRC Press, 1993.

[42] L.M. Malard, M.A. Pimenta, G. Dresselhaus, M.S. Dresselhaus, Raman spectroscopy in graphene, Phys. Rep. 473 (2009) 51-87.

[43] Z. Ni, Y. Wang, T. Yu, Z. Shen, Raman spectroscopy and imaging of graphene, Nano Res. 1 (2010) 273-291.

[44] J.S. Cameron, D.S. Ashley, J.S. Andrew, G.S. Joseph, T.G. Christopher, Accurate thickness measurement of graphene, Nanotechnology 27 (2016) 125704.

[45] F. Bonaccorso, A. Lombardo, T. Hasan, Z. Sun, L. Colombo, A.C. Ferrari, Production and processing of graphene and 2d crystals, Mater. Today 15 (2012) 564-589.

[46] D.C. Marcano, D.V. Kosynkin, J.M. Berlin, A. Sinitskii, Z. Sun, A. Slesarev, L.B. Alemany, W. Lu, J.M. Tour, Improved Synthesis of Graphene Oxide, ACS Nano 4 (2010) 4806-4814.

[47] F. Bonaccorso, A. Bartolotta, J.N. Coleman, C. Backes, 2D-Crystal-Based Functional Inks, Adv. Mater. 28 (2016) 6136-6166.

[48] J.R. Potts, D.R. Dreyer, C.W. Bielawski, R.S. Ruoff, Graphene-based polymer nanocomposites, Polymer 52 (2011) 5-25. 
